\title{
Comparing Eyesi Virtual Reality Simulator and Traditional Teaching Methods for Direct Ophthalmoscopy: Students' Perspectives at Indiana University School of Medicine
}

\author{
Hanna L. Tso, BS ${ }^{1}$ Jason Young, MD ${ }^{1} \quad$ C. W. Yung, MD ${ }^{1}$
}

${ }^{1}$ Department of Ophthalmology, Eugene and Marilyn Glick Eye Institute, Indiana University School of Medicine, Indianapolis, Indiana

J Acad Ophthalmol 2021;13:e66-e72.
Address for correspondence Chi-Wah Yung, MD, Department of Ophthalmology, Eugene and Marilyn Glick Eye Institute, Indiana University School of Medicine, 1160 W. Michigan Street, Indianapolis, IN 46202 (e-mail: ryung@iupui.edu).

\begin{abstract}
Keywords

- medical education

- direct ophthalmoscopy

- Eyesi

- virtual reality simulator
\end{abstract}

Background The fundus examination is an essential part of any ophthalmologic evaluation. However, medical students and primary care physicians often lack confidence with direct ophthalmoscopy. Virtual reality simulators are being employed in medical education to teach this technically challenging examination.

Objective To compare medical student ratings of the Eyesi Direct Ophthalmoscope Simulator and traditional small group teaching methods for learning direct ophthalmoscopy skills.

Methods All medical students at Indiana University School of Medicine traditionally learn direct ophthalmoscopy in their first 2 years during a small group session led by a physician instructor. Students who later enrolled in ophthalmology clinical electives during 2019 and 2020 were invited to additionally complete the Eyesi Direct Ophthalmoscope Simulator virtual reality curriculum. A voluntary, anonymous survey was sent between June and August 2020 to students who had completed both the traditional and Eyesi simulator sessions. Students were asked to rate their confidence in performing direct ophthalmoscopy following each session, and to indicate which teaching method was superior and why. Chi-square analysis was used to compare categorical variables.

Results Students' confidence ratings for performing direct ophthalmoscopy were significantly higher following completion of the Eyesi simulator session compared with the traditional small group session $(p<0.001)$. Four-fifths of respondents felt that the Eyesi simulator was superior to the traditional small group for learning the skills of direct ophthalmoscopy, while one-fifth felt that the two sessions were equally effective $(p<0.001)$. None of the students responded that the small group session was the superior teaching method.

Conclusion The Eyesi Direct Ophthalmoscope Simulator was rated highly among medical students and offers distinct learning advantages that could not be replicated in received

August 23, 2020 accepted after revision February 3, 2021
Dol https://doi.org/ $10.1055 / \mathrm{s}-0041-1726349$ ISSN 2475-4757.

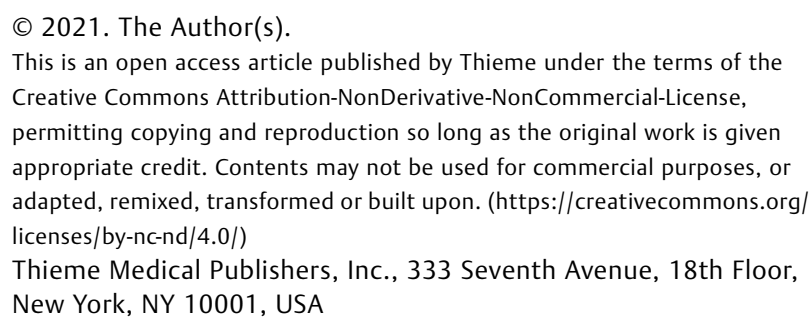
appropriate credit. Contents may not be used for commercial purposes, or adapted, remixed, transformed or built upon. (https://creativecommons.org/ licenses/by-nc-nd/4.0/) Thieme Medical Publishers, Inc., 333 Seventh Avenue, 18th Floor, New York, NY 10001, USA 
a traditional small group environment, such as providing numerous examples of pathological findings and allowing unlimited examination time without concern for patient's inconvenience or light exposure. The Eyesi simulator is a promising tool for teaching direct ophthalmoscopy to medical students. Ultimately, familiarity with the fundus examination will enable future physicians across specialties to better evaluate and appropriately refer patients with ocular fundus pathology.

The fundus examination is an essential part of any ophthalmologic evaluation. While ophthalmologists routinely examine the fundus, primary care physicians (PCPs), emergency medicine physicians, and neurologists are often the first to assess patients for visual symptoms or ocular manifestations of systemic diseases. Given the prevalence of blinding conditions such as age-related macular degeneration and diabetic retinopathy, timely referral of patients by first-line providers to ophthalmologists for further evaluation and management is vital. ${ }^{1,2}$ Therefore, it is important for physicians other than ophthalmologists to feel comfortable performing a fundus examination with a direct ophthalmoscope.

However, the funduscopic examination appears to be a dying art. $^{3}$ It is often omitted due to technical difficulty, waning enthusiasm for ophthalmoscopy, and even discouragement from preceptors in medical education. ${ }^{3-5}$ Multiple studies have demonstrated a lack of confidence in direct ophthalmoscopy among medical students and PCPs. ${ }^{3,6-9}$ As clinicians other than ophthalmologists typically only have a direct ophthalmoscope at their disposal, comfort and confidence with direct ophthalmoscopy are likely the determining factors for these physicians to perform a fundus examination. Studies have shown that few clinicians perform direct ophthalmoscopy, and many who do are unable to reliably detect abnormalities of the ocular fundus. ${ }^{3}$ Ultimately, comfort with this technique stems from appropriate undergraduate medical education. ${ }^{7}$

Traditionally, medical students in their first 2 years at Indiana University School of Medicine (IUSM) are taught to use the direct ophthalmoscope during a small group session led by a physician. The instructor demonstrates the examination technique, then students briefly examine either a standardized patient's or each other's eyes. However, the instructor may or may not be an ophthalmologist or be proficient in direct ophthalmoscopy. Furthermore, the instructor cannot reliably verify which structures students have visualized. As instructors are unable to objectively assess students' performance and accuracy with the ophthalmoscope, they must rely on students' self-reports which may not be reliable.

Recently, virtual reality simulators for direct ophthalmoscopy have been employed in medical education. Multiple studies have shown that simulators can be effective tools to practice the fundus examination. ${ }^{10-14}$ At this time, however, few studies have assessed the individual simulators. ${ }^{11-13}$

One simulator of special interest is the Eyesi Direct Ophthalmoscope Simulator (VRmagic Holding AG, Man- nheim, Germany). ${ }^{15}$ Students use the provided direct ophthalmoscope to examine the eyes of a mannequin face, which allows them to visualize a sample retina in virtual reality (-Fig. 1). The simulator's curriculum consists of four modules that progress in difficulty. Students are introduced to basic device handling, identification of abstract findings on the retina, examples of healthy and diseased retinas, and finally, documentation of common pathological findings. The simulator offers a platform for standardized, self-guided training of large classes. ${ }^{15}$ A previous study found strong evidence of validity for the Eyesi simulator's ability to distinguish between expert and novice performance. ${ }^{13}$ Experienced ophthalmologists significantly outperformed novices, and the pass/fail standard showed excellent discriminatory ability with no false positives or negatives.

The Eyesi Direct Ophthalmoscope Simulator was recently acquired by the Department of Ophthalmology, IUSM and employed as an adjunct to the traditional small group curriculum for medical students enrolled in ophthalmology clinical electives. In this study, we aimed to evaluate medical students' perspectives on the Eyesi simulator, as compared with the traditional small group session, for improving comfort and confidence in performing direct ophthalmoscopy.

\section{Methods}

To compare the traditional small group and Eyesi simulator sessions for direct ophthalmoscopy, we surveyed IUSM medical students who had completed both teaching methods to ask about their experiences with each and to ask which one they felt was superior for learning.

\section{Survey Development}

A survey consisting of nine questions was created on Google Forms (Google LLC, Mountain View, CA). The questions asked the students to rate their comfort and confidence in performing direct ophthalmoscopy on a live patient following their small group session versus their Eyesi simulator session (for a copy of the survey, see Supplementary Appendix). It also asked the students whether they felt that the Eyesi simulator or small group session was the superior teaching method and to comment on why.

\section{Sample}

We administered the survey to 58 IUSM medical students who had learned direct ophthalmoscope skills through both 


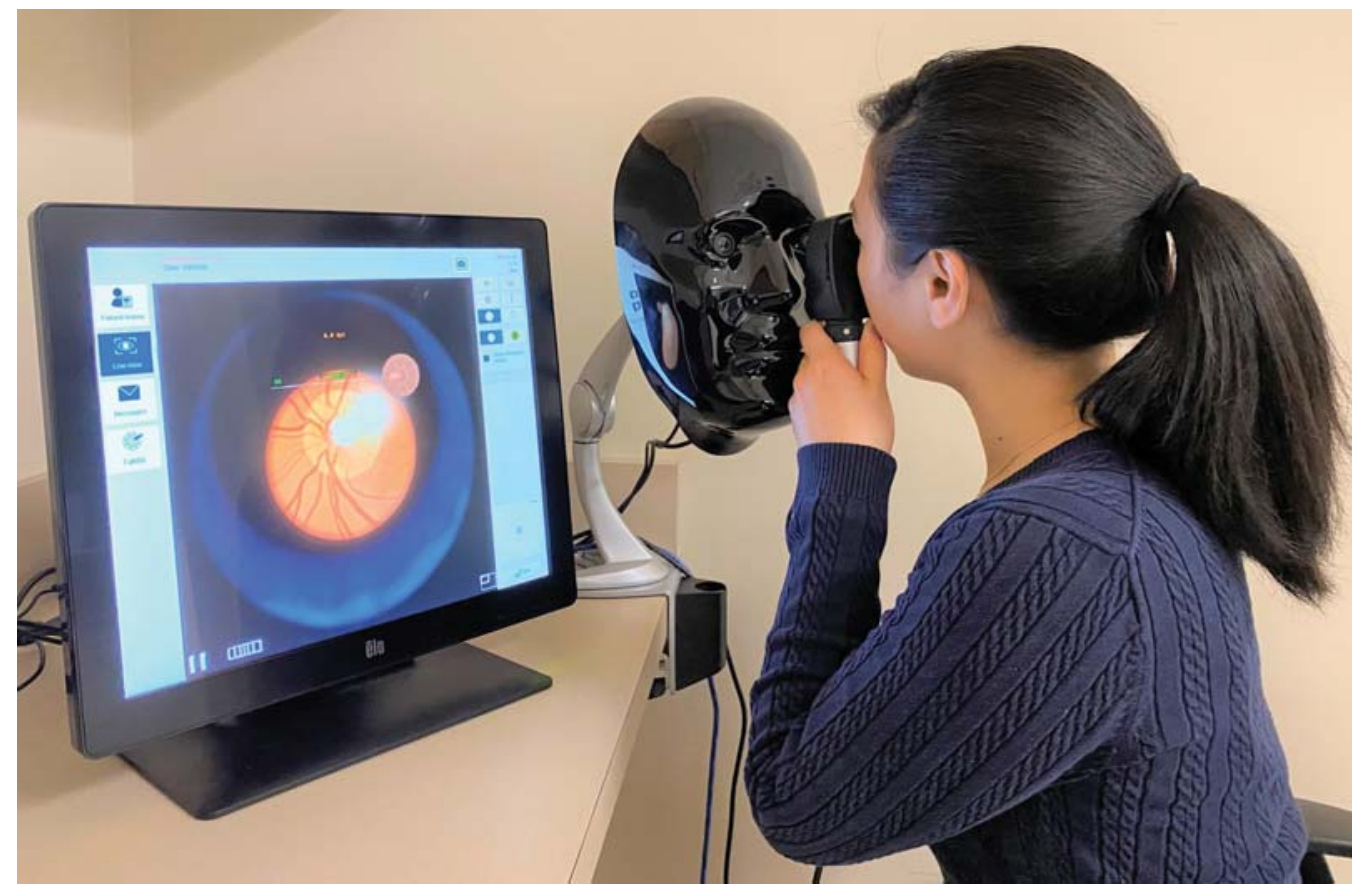

Fig. 1 Student using the Eyesi Direct Ophthalmoscope Simulator to examine a virtual patient. Computer monitor displays student's view of the fundus in virtual reality.

the traditional small group curriculum during their first 2 years and later the Eyesi Direct Ophthalmoscope Simulator during an ophthalmology clinical elective. Only students who had completed all four Eyesi training modules were included. Invitations to participate in the survey were sent to the students' IUSM e-mail addresses, and participation in the survey was voluntary and anonymous. The IUSM's Institutional Review Board and committees on human subjects granted exempt status for this study.

\section{Data Analysis}

We calculated the response rate for medical students using 58 as the denominator. Chi-square analysis was used to compare categorical variables. For all analyses, $p<0.05$ was considered statistically significant.

\section{Results}

A total of 31 medical students participated in the survey for a response rate of $53.4 \%(31 / 58)$. The majority of student respondents completed the Eyesi virtual reality simulator session during their third year of medical school $(23 ; 74.2 \%)$ ( - Table 1). More than half of the respondents completed their traditional small group session on the main IUSM campus in Indianapolis during the first 2 years of medical school (19; $61.3 \%$ ), whereas others were from five of the eight regional campuses across Indiana (12; 38.7\%). Of note, only a minority of small group sessions were led by ophthalmologists ( 5 ; $16.1 \%)$. During these sessions, most students used a traditional direct ophthalmoscope (26; 83.9\%). Several students opted to use a PanOptic ophthalmoscope $(5 ; 16.1 \%)$.

On a scale of 1 to 5 , students were asked to rate how comfortable and confident they felt in performing direct
Table 1 Medical students' perspectives on learning direct ophthalmoscopy skills through the Eyesi virtual reality simulator and traditional small group sessions

\begin{tabular}{|c|c|}
\hline \multicolumn{2}{|c|}{ Year in medical school when you used the Eyesi simulator, $n(\%)$} \\
\hline 2 & $1(3.2)$ \\
\hline 3 & $23(74.2)$ \\
\hline 4 & $7(22.6)$ \\
\hline \multicolumn{2}{|c|}{ Indiana University School of Medicine campus, $n$ (\%) } \\
\hline Indianapolis (main campus) & $19(61.3)$ \\
\hline West Lafayette & $5(16.1)$ \\
\hline Fort Wayne & $3(9.7)$ \\
\hline Bloomington & $2(6.5)$ \\
\hline South Bend & $1(3.2)$ \\
\hline Terre Haute & $1(3.2)$ \\
\hline \multicolumn{2}{|c|}{$\begin{array}{l}\text { Small group session for learning the direct ophthalmic } \\
\text { examination was led by an ophthalmologist, } n(\%)\end{array}$} \\
\hline Yes & $5(16.1)$ \\
\hline No & $24(77.4)$ \\
\hline I do not know & $2(6.5)$ \\
\hline \multicolumn{2}{|c|}{$\begin{array}{l}\text { Type of ophthalmoscope used during small group } \\
\text { session, } n(\%)\end{array}$} \\
\hline Traditional direct ophthalmoscope & $26(83.9)$ \\
\hline PanOptic ophthalmoscope & $5(16.1)$ \\
\hline \multicolumn{2}{|c|}{$\begin{array}{l}\text { Teaching method you feel is superior for learning direct } \\
\text { ophthalmoscopy skills, } n \text { (\%) }\end{array}$} \\
\hline Eyesi direct ophthalmoscope simulator & $25(80.6)$ \\
\hline Traditional small group session & $0(0)$ \\
\hline $\begin{array}{l}\text { Eyesi simulator and small group training } \\
\text { sessions are equally effective }\end{array}$ & $6(19.4)$ \\
\hline
\end{tabular}




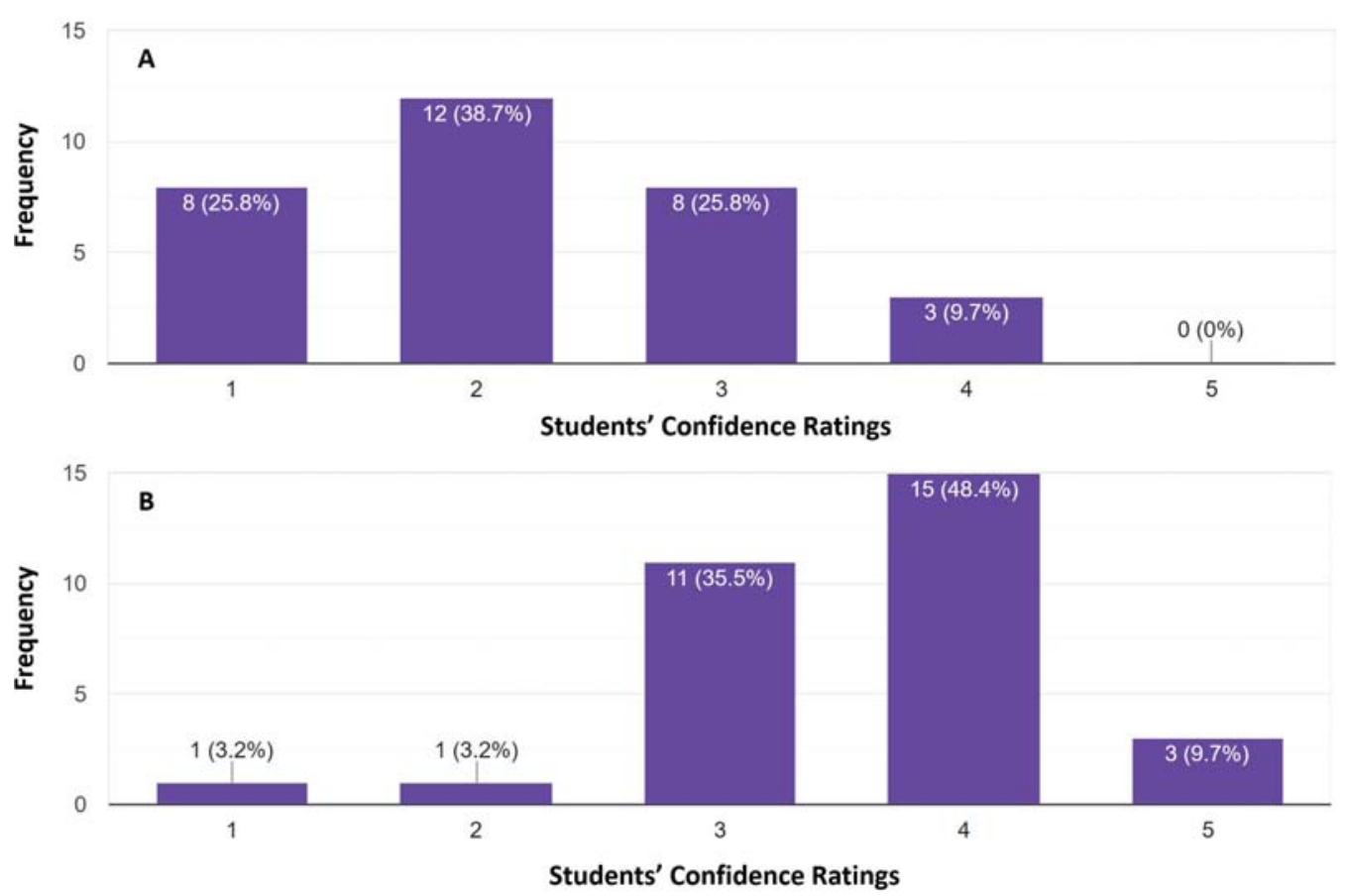

Fig. 2 Medical students' ratings of comfort and confidence in performing direct ophthalmoscopy on a live patient following the (A) traditional small group training session and (B) Eyesi virtual reality simulator session. A rating of 1 indicated that the student was not at all comfortable or confident, while a rating of 5 indicated that the student was very comfortable and confident. The confidence ratings of students following the Eyesi simulator session (mean $=3.58 / 5.0)$ were significantly higher than those following the small group session $($ mean $=2.19 / 5.0)(p<0.001)$.

ophthalmoscopy on a live patient following their small group and Eyesi sessions. A rating of 1 indicated that the student was not at all comfortable, while a rating of 5 indicated that the student was very comfortable. The confidence ratings of students following the Eyesi simulator session (mean $=3.58 / 5.0$ ) were significantly higher than those following the small group session $($ mean $=2.19 / 5.0) \quad(p<0.001)$ (-Fig. 2). In other words, respondents felt more comfortable and confident in performing direct ophthalmoscopy on a live patient following their Eyesi session as compared with their small group session.

Following the small group sessions, students who had been taught by ophthalmologists reported slightly higher confidence ratings (mean $=2.4 / 5.0$ ) than those taught by physicians of another specialty (mean $=2.13 / 5.0$ ). However, the difference in confidence ratings was not statistically significant $(p=0.489)$.

Four-fifths of respondents felt that the Eyesi simulator was superior to the traditional small group for learning the skills of direct ophthalmoscopy $(25 ; 80.6 \% ; p<0.001)$ (-Table 1). One-fifth of students felt that the two teaching methods were equally effective $(6 ; 19.4 \% ; p<0.001)$. None of the students responded that the small group session was the superior teaching method.

The students' free-response comments elucidated why the Eyesi simulator was the more popular teaching method. The most commonly cited strength was that Eyesi provided examples of ocular pathology. Respondents emphasized that this pathology could not be visualized during a small group session with healthy students or volunteers. Additionally, students greatly appreciated the unlimited practice time with the ophthalmoscope. As the user practices on a mannequin, he or she need not worry about patient's comfort, awkwardness, or light exposure while initially learning the examination technique. One student noted that this repetitive practice for hours would be impossible to replicate even with the most patient of volunteers during a small group session.

Other cited strengths of Eyesi included its guidance during the examination (e.g., real-time labeling of pathological findings in virtual reality) and its modules on proper documentation of pathology. Students welcomed the opportunity to identify physiologic structures of the fundus, which prepared them to examine real patients. Furthermore, they appreciated the highly realistic virtual simulation, the opportunity to improve hand-eye coordination with the ophthalmoscope, and the stepwise progression of difficulty. Students felt that Eyesi was a better, easier starting point for learning the basics of the fundus examination as compared with learning on a live volunteer. In general, students seemed to like the low-stakes virtual reality training session, which helped them develop proper technique for visualizing the fundus without concern for patient's inconvenience. Finally, students valued the objective metrics and measurable goals of Eyesi (e.g., percentage of fundus visualized, total light exposure time, and identification of pathology), which could not be reliably monitored during a subjective small group session. A couple students commented that the combined practice with the Eyesi training and an ophthalmology clinical rotation was helpful in further building confidence with the fundus examination.

Several students noted that the Eyesi modules were lengthy. Based on performance data for students who may 
or may not have participated in our survey, the average completion time for the four Eyesi modules was 3.9 hours with a range of 1.8 to 7.4 hours. Several students noted that they experienced eye fatigue and neck or back soreness due to the prolonged positioning. In addition, despite the breadth of pathological examples, one student wished for more baseline examples of a "normal" fundus. A couple students desired faculty guidance during the Eyesi session. One student commented that the small group session was more natural and better replicated the examination in actual practice. Another commented that the Eyesi training was only useful in conjunction with a small group session.

\section{Discussion}

Our results demonstrate that, on average, medical students felt more comfortable and confident in performing direct ophthalmoscopy on a live patient following their Eyesi virtual reality simulator session, as compared with the traditional small group session. Of the respondents, $80.6 \%$ felt that the Eyesi simulator was the superior training method, and $19.4 \%$ felt that the two teaching methods were equally effective. These findings make a strong case for the incorporation of the Eyesi Direct Ophthalmoscope Simulator in medical education.

The free-response comments written by surveyed medical students provided insight into the important advantages of Eyesi. Students appreciated the ability to view many fundus examples, including those with ocular pathology, the unlimited practice time with the ophthalmoscope, the lack of patient's inconvenience or light exposure, the objective measurable goals, and the real-time labeling of physiologic structures and pathology in virtual reality. These advantages could not be replicated in a small group session, no matter how experienced the instructor or how patient the volunteers.

One student praised Eyesi for its stepwise progression of difficulty. The Eyesi simulator consists of four modules which sequentially guide students through the fundus examination. First, students learn about basic device handling, including the diopter settings and light intensity. Next, they learn to maneuver with the ophthalmoscope to identify abstract findings on the retina, such as squares or circles. They then view examples of healthy retinas and pathological findings. The final module culminates in a testing mode that asks students to systematically identify and document pathology. In contrast, the examination of a patient in a clinic or a volunteer in a small group session is difficult to break down into discrete steps. In these settings, inexperienced learners are immediately expected to handle the ophthalmoscope, view the retina, and report back on their findings. Thus, Eyesi makes the fundus examination less daunting for students by enabling them to progress at their own pace with a suitable level of challenge and immediate feedback.

Another distinct advantage of the Eyesi simulator is the potential for standardized education. As evidenced by the survey findings, the majority of traditional small group teaching sessions were not led by ophthalmologists. The lack of uniformity among small group instructors and their teaching styles raises the question of how standardized small group sessions are. Moreover, it is concerning that the instructors teaching students may not be very comfortable with ophthalmoscopy themselves. This gap in standardized education is particularly relevant to IUSM. As one of the largest medical schools in the United States, IUSM trains more than 350 medical students per class-year across nine campuses in Indiana. The variability in instruction across campuses impedes uniform benchmarks for direct ophthalmoscopy skills. Other medical schools with large class sizes may face similar issues. By providing a consistent method of instruction, the Eyesi simulator provides a unique opportunity for IUSM and other medical schools to adopt a standard curriculum for their students for the direct ophthalmoscope examination.

Additionally, Eyesi is a useful tool for providing objective metrics of students' performance, including the percentage of the fundus viewed, the total light exposure time, and the number of pathological findings identified. In contrast, the small group session is inherently limited in that instructors, no matter how experienced, cannot verify which parts of the fundus a student has visualized. Students lacking familiarity with the physiologic structures of the fundus may struggle to report their findings. To address this issue, each Eyesi module provides a "map" of a fundus and records which structures a user has viewed, such as the optic nerve and macula. In particular, one student commented that the simulator allowed them to appreciate how little of the fundus he or she was visualizing at first.

Given this instantaneous feedback, the self-guided modules of Eyesi are conducive to remote learning. Students need not depend on patients or volunteers to practice direct ophthalmoscopy, but rather can practice independently with the Eyesi simulator and track their own performance. Moreover, educators can monitor students' progress remotely by accessing training data through an educator web portal. Eyesi allows educators to track objective metrics including the number of cases completed by a student, the pathological findings identified, and the time spent on each case. Instructors can even view statistics including performance scores, class rankings, and training performance comparisons among students. They can utilize this information to provide additional support to struggling individuals. These features would greatly assist an instructor in keeping track of numerous medical students, even with large class sizes.

The self-guided learning of Eyesi can be viewed as an advantage, as students can progress through the modules at their own pace. However, the independent learning requires that only one student use the simulator at a given time. At IUSM, students are asked to reserve a time slot to use the simulator so that multiple students do not overlap. Medical schools with large class sizes may struggle to accommodate enough time sessions for all students, even though these are the very schools that would benefit the most from a standardized ophthalmoscopy curriculum. This issue could be mitigated if an institution acquired several simulator units to allow for multiple, simultaneous practice sessions. However, 
due to the high cost of the Eyesi unit relative to other simulators, medical schools would need to optimize their student arrangement within financial limitations.

One student expressed the desire for faculty guidance during the Eyesi simulator session to ensure proper technique and to troubleshoot any issues. The Eyesi setup is designed for self-paced learning by the student, and the presence of an instructor is an option. In fact, Eyesi would facilitate assistance by an educator, as the simulator projects the student's view of the fundus onto a computer screen, which can be viewed by others simultaneously. Seeing the student's view, the educator could then help the student troubleshoot. This level of in-depth feedback and support would not be possible in a small group, even with an experienced instructor. Furthermore, instructors could remotely monitor students' progress and selectively provide additional support to struggling students.

Some students observed that the Eyesi curriculum was lengthy. Given that the average completion time for the four training modules was 3.9 hours, several respondents complained of eye fatigue and back or neck soreness by the end. Interestingly, the length of the Eyesi training could be viewed as an advantage. Mastering direct ophthalmoscopy requires ample practice time to build hand-eye coordination and reliably visualize structures of the fundus. Small group sessions may be limited in length due to instructor's availability or volunteer discomfort from light exposure, thereby reducing practice time for students. In contrast, the objective scoring by the Eyesi simulator forces students to demonstrate proficiency at one level before advancing to subsequent modules. Therefore, despite the rigorous training, instructors can have confidence in students' skills upon completion of the Eyesi curriculum. In the future, the ergonomic issues could be resolved by encouraging students to break up the Eyesi training into multiple sittings to make it more manageable.

Another suggestion to reduce the time requirement would be for medical schools to require that preclinical students complete only the first two Eyesi modules which teach basic direct ophthalmoscopy skills, including device handling and identification of abstract findings on the retina. The average time for our surveyed students to complete both modules was 56 minutes. Later, during ophthalmology clinical electives, more motivated students could complete the final, more time-consuming modules.

Finally, some students were concerned that the simulator did not feel natural, did not replicate the need to position a live patient, did not offer a variety of "baseline normal" fundus images, and required the small group session in conjunction to be useful. Addressing these concerns, an ideal medical curriculum would include both the Eyesi and small group sessions. For instance, students could first use the Eyesi simulator to gain comfort in handling the ophthalmoscope, identifying physiologic structures of the fundus, and recognizing "baseline" healthy fundus appearances, before moving on to the small group session to gain experience with positioning a volunteer, limiting light exposure time, and appreciating physiologic variants of the fundus. Such a two- pronged approach would provide a strong primer for students before examining patients in the clinic setting. Based on students' feedback, rather than using the Eyesi simulator to replace small group sessions as the sole teaching method, we advocate for Eyesi to be used as a complementary modality.

This study has several limitations. As the survey was conducted retrospectively, recall bias could have prevented students from accurately recalling and rating their confidence in performing direct ophthalmoscopy following each teaching session. For future studies, students would ideally be surveyed immediately after each session.

Additionally, the study may have experienced selection bias in favor of Eyesi if surveyed students who had greater interest in Eyesi were more likely to respond and rate it highly. In particular, students planning to pursue ophthalmology as a specialty may have been highly motivated and engaged during both teaching sessions, leading to higher confidence ratings overall for that subgroup of students compared with others. However, this bias was likely limited as only $13.8 \%(8 / 58)$ of the surveyed students expressed interest in an ophthalmology career.

Moreover, all students completed the Eyesi simulator training after the small group session. An important consideration is that students may have felt more confident after completing the Eyesi training simply because they had additional practice after the initial small group sessionboth from Eyesi and potentially during ophthalmology clinical rotations. For this reason, the positive ratings and reviews of Eyesi may have been artificially inflated. To limit bias due to the sequence of instruction, future studies would ideally ask one group of students to complete the small group session first and another group to complete the Eyesi session first. Notably, a typical small group session might last for half an hour or less, whereas the Eyesi modules require several hours to complete (mean $=3.9$ hours). It is unclear whether students' improved confidence following the Eyesi session was due to the efficiency of the teaching method or simply due to the significantly longer practice time.

Furthermore, these survey results relied on subjective student ratings of the Eyesi and small group teaching methods. This study was designed to seek students' subjective feedback, but not to objectively measure the impact of Eyesi on students' skills. To quantify increases in students' skill levels, future studies could implement skill assessments as pre- and posttests for each teaching session. One objective assessment that has been described would involve students examining five standardized patients with distinct ocular findings. ${ }^{14}$ Students would be asked to calculate the cup-todisc ratio, comment on the disc margins, describe any macular pathology, and attempt to match unlabeled fundus photographs to the corresponding patients.

Despite the standard method of instruction by the Eyesi simulator, this study is limited due to the variable delivery of the small group sessions. Across the nine IUSM campuses, the small group sessions would have been led by different physician instructors with varying expertise and teaching styles, varying numbers of students, and varying lengths of 
the sessions. Thus, each campus likely had a different definition of a "small group session." Our survey did not explore these differences beyond inquiring whether or not the instructor was an ophthalmologist. We also did not ask for the instructor's specialty if not ophthalmology. The range in quality of instruction would have impacted students' confidence with direct ophthalmoscopy following the small group session, which could explain the wide range of confidence ratings from the survey. Interestingly, however, despite the lack of uniformity across campuses, students' confidence ratings following the Eyesi session were still significantly higher than those following the small group session $(p<0.001)$ (-Fig. 2 ). This finding would support the acquisition of an Eyesi simulator for each campus to standardize the delivery of instruction for the direct ophthalmoscope examination and ultimately improve students' confidence.

Finally, the modest sample size in this study may limit the confidence of the chi-square statistical analysis and the conclusions drawn. We plan to continue surveying IUSM medical students who complete both the small group and Eyesi training sessions to grow our sample size. Future studies could ask more detailed questions about the small group sessions (e.g., instructor's specialty, length of session, and number of students). They could also gather students' feedback about how to best integrate Eyesi into the medical school curriculum, such as by making the Eyesi session a prerequisite for the small group session and by spreading out the Eyesi modules over multiple sittings to make them more manageable.

\section{Conclusion}

In summary, our findings support the implementation of the Eyesi Direct Ophthalmoscope Simulator in medical education. The Eyesi simulator was rated highly among IUSM medical students and offers distinct advantages that could not be replicated in a traditional small group environment. Our findings demonstrate great promise in employing this virtual reality technology to teach a historically technically challenging examination. We encourage medical schools to consider adding the Eyesi or similar simulators to their ophthalmology curriculum as tools for improving student proficiency and confidence with direct ophthalmoscopy. Although medical students pursuing ophthalmology would certainly benefit from familiarity with ophthalmoscopy, students pursuing primary care specialties who will frequently be the first to evaluate patients with visual symptoms may benefit the most from simulator training. The ability of future physicians to confidently perform fundus examinations will ultimately translate into better patient care and improved outcomes.

Conflict of Interest

None declared.

\section{References}

1 World Health Organization. Causes of blindness and visual impairment. 2020. Accessed August 6, 2020 at: https://www.who.int/ news-room/fact-sheets/detail/blindness-and-visual-impairment

2 Wong WL, Su X, Li X, et al. Global prevalence of age-related macular degeneration and disease burden projection for 2020 and 2040: a systematic review and meta-analysis. Lancet Glob Health 2014;2(02):e106-e116

3 Mackay DD, Garza PS, Bruce BB, Newman NJ, Biousse V. The demise of direct ophthalmoscopy: a modern clinical challenge. Neurol Clin Pract 2015;5(02):150-157

4 Rodenbeck SJ, Mackay DD. Examining the ocular fundus in neurology. Curr Opin Neurol 2019;32(01):105-110

5 Kelly LP, Garza PS, Bruce BB, Graubart EB, Newman NJ, Biousse V. Teaching ophthalmoscopy to medical students (the TOTeMS study). Am J Ophthalmol 2013;156(05):1056-1061.e10

6 Mackay DD, Garza PS, Bruce BB, et al. Teaching ophthalmoscopy to medical students (TOTeMS) II: a one-year retention study. Am J Ophthalmol 2014;157(03):747-748

7 Stern GAThe Association of University Professors of Ophthalmology Education Committee. Teaching ophthalmology to primary care physicians. Arch Ophthalmol 1995;113(06):722-724

8 Gupta RR, Lam WC. Medical students' self-confidence in performing direct ophthalmoscopy in clinical training. Can J Ophthalmol 2006;41(02):169-174

9 Shuttleworth GN, Marsh GW. How effective is undergraduate and postgraduate teaching in ophthalmology? Eye (Lond) $1997 ; 11(\mathrm{Pt}$ 5):744-750

10 Ricci LH, Ferraz CA. Ophthalmoscopy simulation: advances in training and practice for medical students and young ophthalmologists. Adv Med Educ Pract 2017;8:435-439

11 Bukhari AA. The clinical utility of eye exam simulator in enhancing the competency of family physician residents in screening for diabetic retinopathy. Saudi Med J 2014;35(11): 1361-1366

12 Boden KT, Rickmann A, Fries FN, et al. Evaluation of a virtual reality simulator for learning direct ophthalmoscopy in student teaching [in German]. Ophthalmologe 2020;117(01):44-49

13 Borgersen NJ, Skou Thomsen AS, Konge L, Sørensen TL, Subhi Y. Virtual reality-based proficiency test in direct ophthalmoscopy. Acta Ophthalmol 2018;96(02):e259-e261

14 Bénard-Séguin É, Kwok J, Liao W, Baxter S. Use of a fundus photograph matching program in imparting proficiency in ophthalmoscopy. Can J Ophthalmol 2018;53(05):480-485

15 VRmagic. Eyesi direct. 2020. Accessed August 6, 2020 at: https://www. vrmagic.com/medical-simulators/Eyesi-direct-ophthalmoscope 